\title{
Analyse de l'évolution du système pastoral du Maroc oriental
}

\author{
A. Bechchari ${ }^{1 *}$ A. El Aich ${ }^{2}$ H. Mahyou ${ }^{1}$ \\ M. Baghdad ${ }^{2}$ M. Bendaou ${ }^{1}$
}

\begin{abstract}
Mots-clés
Ovin - Parcours - Dégradation des terres - Pastoralisme - Politique de
\end{abstract}

\section{Résumé}

Le système pastoral des hauts plateaux du Maroc oriental est caractérisé par l'étendue des parcours (3,2 millions d'hectares) et la dominance de l'élevage ovin (1,2 million de têtes). Ce travail, mené à Béni Mathar au nord et à Maâtarka au sud, traite la question des changements d'occupation des terres de parcours et de conduite des troupeaux, à partir de l'hypothèse d'une dégradation des parcours suivie d'une baisse de la pression de pâturage. Cette dynamique est perçue par l'étude de l'occupation des sols en 1970, 1988 et 2013. Les modes de conduite sont identifiés au travers d'enquêtes (85) et lors d'ateliers participatifs. L'analyse de l'occupation des sols montre la régression des bonnes formations végétales et leur substitution par des formations de moindre valeur, et une baisse des apports fourragers sur parcours. Parallèlement, on note une augmentation des effectifs d'animaux de moins en moins mobiles et de plus en plus supplémentés (20 à 40 p. 100 de satisfaction des besoins, essentiellement par l'orge et le son de blé), et un changement progressif dans la composition raciale des troupeaux en faveur de la race Ouled Djellal (à valeur commerciale compétitive) au détriment de la race locale Béni Guil. L’histoire de la société pastorale étudiée témoigne d’une capacité d'adaptation malgré les perturbations socio-économiques et climatiques relevées depuis I'indépendance. Les grands éleveurs (plus de 200 brebis) profitent davantage des pâturages et savent mieux spéculer lors de la commercialisation que les petits éleveurs (moins de 50 brebis) qui sont davantage menacés par l'abandon de l'élevage.

\section{INTRODUCTION}

L'activité pastorale au Maroc oriental est au centre de nombreux enjeux. Les aspects sociopolitiques ont dicté, depuis le début du $\mathrm{XX}^{\mathrm{e}}$ siècle, la fixation des populations autrefois nomades. La dégradation des parcours steppiques de la zone s'est accentuée au fil des années donnant lieu à une perte de biomasse et à l'altération de la biodiversité des espèces végétales.

L'aridité du milieu et la fréquence élevée des années de sécheresse comptent parmi les facteurs responsables de la faible productivité et de l'affaiblissement de la capacité de régénération du couvert

1. Institut national de la recherche agronomique, BP 415 RP, Rabat, Maroc.

2. Institut agronomique et vétérinaire Hassan II, Rabat Instituts, Rabat, Maroc.

* Auteur pour la correspondance

Tél. : +212 (0)6 67241034

E-mail : bechchari@yahoo.com végétal sur un sol pauvre et très vulnérable à l'érosion. L'abandon progressif de la mobilité des troupeaux et leur sureffectif, ainsi que l'extension des terres de culture sont autant de facteurs qui accélèrent les processus de dégradation de la végétation (23).

Dans cette étude, focalisée sur les communes de Béni Mathar au nord et de Maâtarka au sud, nous avons analysé l'évolution du système pastoral de la zone des hauts plateaux du Maroc oriental, communément connu pour l'élevage ovin sur de vastes étendues de parcours collectifs. La dynamique des principales formations pastorales de la zone a été abordée pour tenter de déterminer si les stratégies suivies par les éleveurs tiennent compte de la dynamique de la végétation exploitée. Des données sur la population de ces communes sont présentées dans le tableau I.

Devant les projections climatiques défavorables, comme la récurrence des années de sécheresse (22) qui ne permettent pas la restauration des ressources pastorales sous pression animale croissante, les hypothèses suivantes ont été émises : a) la dynamique de 


\section{Tableau I}

Population dans la zone d'étude située dans I'Oriental au Maroc en 2010

\begin{tabular}{|c|c|c|c|c|c|}
\hline & Habitants & 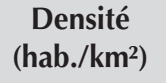 & $\begin{array}{c}\text { Accroissement } \\
(\%)\end{array}$ & $\begin{array}{c}\text { Ménages } \\
\text { (nb. de personnes) }\end{array}$ & $\begin{array}{c}\text { Pauvreté } \\
(\%)\end{array}$ \\
\hline Béni Mathar & 7078 & 2 à 5 & 3,1 & $1152(6)$ & 37 \\
\hline Maâtarka & 8030 & $<1$ & $-0,7$ & $1125(7)$ & 62,5 \\
\hline
\end{tabular}

la végétation s'accompagne de la régression des bonnes formations végétales en faveur d'espèces de moindre valeur fourragère, et b) la dégradation des ressources végétales, en affectant l'offre fourragère sur parcours, conduit à la baisse des effectifs ovins.

Jusqu'au début du $\mathrm{XX}^{\mathrm{e}}$ siècle, la nature du milieu et la complémentarité entre les différents secteurs de la zone avaient toujours orienté les populations vers un mode d'exploitation des ressources basé sur le nomadisme (13). La zone était qualifiée de zone pastorale à élevage vraiment extensif jusqu'à l'occupation par les troupes françaises en 1904, date d'un pacte avec les notables de Béni Mathar. La société pastorale des hauts plateaux est passée, en quelques années seulement, d'une société nomade (ou du moins d'une société où le déplacement occupe une place importante) à une société fixée sur des aires de pâturage, sur lesquelles les amplitudes de déplacement étaient de plus en plus restreintes (19), limitées à une dizaine de kilomètres du campement.

Les signes de destruction de la société pastorale remontent aux années 1920 quand plusieurs facteurs ont progressivement transformé le nomadisme de la zone. On note, entre autres, le problème de la sécurité, la création de nouveaux marchés largement tournés vers l'exportation, l'ouverture des mines et des chantiers de l'alfa, la démographie, des changements dans les formes de solidarité, l'expansion de l'agriculture, l'effet des aléas climatiques. Les communautés pastorales de base (réseaux de relations sociales effectives ou douars [groupements d'habitations] [29]), qui avaient l'habitude de se déplacer en groupe défensif et de camper ensemble, ne le font plus depuis les années 1940.

Après la sécheresse de 1945-46 et ses effets néfastes sur les troupeaux (beaucoup de troupeaux décimés), les années 1950 ont marqué le début du nomadisme de petits groupes et l'apparition du camion chez les plus gros éleveurs aux dépens du dromadaire qui recule alors progressivement. C'est à cette époque qu'on note un grand nombre de petits éleveurs qui vivaient sur un parcours d'élevage à rayon court, l'importance du travail salarial temporaire (dépendance d'une grande partie de la population par rapport aux nouveaux revenus provenant du travail dans les mines, les chantiers d'alfa et l'émigration), et le début de la double implantation steppe/ville donnant lieu à une vie bipolaire (rurale et urbaine). La mobilité spatiale n'est plus qu'une alternative (arrêt des déplacements) vers les années 1960.

La société pastorale, très déstructurée à l'aube de l'indépendance (1956), s'est reconstruite, surtout après les années 1990, sur des bases nouvelles différentes de celles d'autrefois. Ainsi, environ 67 p. 100 des tentes et des troupeaux étaient encore installés en milieu rural, 22 p. 100 des familles vivaient en ville et 10 p. 100 avaient émigré. Ce rétablissement témoigne d'une capacité de réadaptation dictée par l'importance de l'activité d'élevage sur les vastes étendues de parcours.

A présent, la régression des apports fourragers des parcours et la tendance à la désertification ont favorisé l'émergence de nouveaux systèmes d'élevage plus sédentaires et tributaires de la supplémentation. De plus, la recherche d'une diversification des sources de revenus pour pallier les incertitudes du milieu ont fait apparaitre des comportements individuels opportunistes et des modes de vie bipolaires.

\section{MATERIEL ET METHODES}

L'étude concerne le système pastoral des hauts plateaux du Maroc oriental, reconnus pour leurs vastes faciès pastoraux (plus de trois millions d'hectares de pâturages) et l'activité dominante de l'élevage ovin sur parcours, conduit en mode extensif (environ 1,6 million de têtes).

\section{Zone d'étude}

Les communes de l'étude (figure 1) ont été sélectionnées en raison des différences de dégradation des ressources végétales supposées être plus fortes à Béni Mathar au nord qu'à Maâtarka au sud où

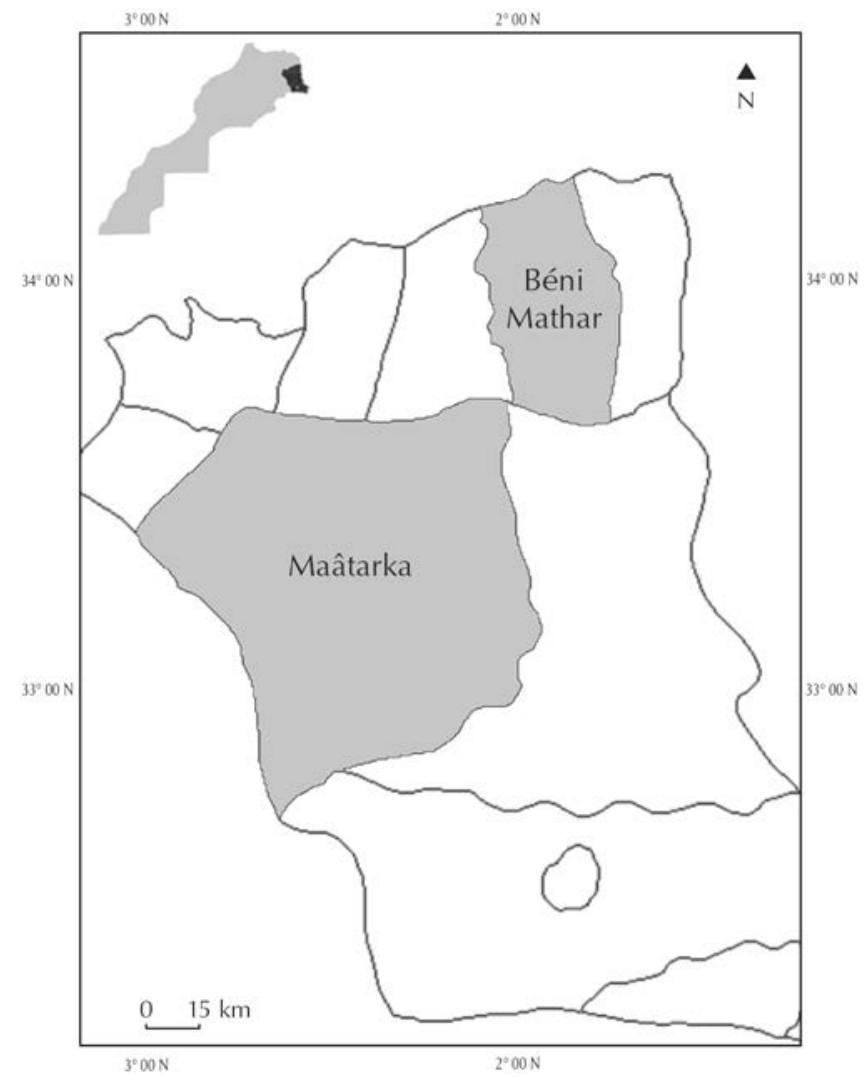

Figure 1 : carte montrant les deux communes de l'étude situées dans les hauts plateaux de l'Oriental au Maroc. 
les espaces de pâturage sont plus étendus. Les éleveurs de Béni Mathar, plus sédentaires, exploitent un espace de $1690 \mathrm{~km}^{2}$ environ avec un effectif de 83830 têtes, alors que ceux de Maâtarka exploitent un espace pastoral plus vaste de $6980 \mathrm{~km}^{2}$ environ avec un effectif de 272210 têtes (tableau II).

Les systèmes d'élevage pratiqués sont de type pastoral extensif à semi-extensif. L'élevage, principale activité de la zone, se caractérise par une productivité annuelle faible de 14 à 18 kilogrammes par unité zootechnique (soit une brebis et sa suite), suite aux contraintes du milieu et à la conduite traditionnelle des éleveurs. Dominé par l'espèce ovine (tableau II), l'élevage tire profit des pâturages naturels de la zone et de la supplémentation en concentré (à base d'orge et de son) qui est devenue courante après les années 1980, malgré sa nette dépendance aux marchés et souks locaux.

Les terrains de parcours, globalement de statut collectif, sont ouverts à tous les usagers d'une même tribu avec une tendance au cantonnement à un niveau infratribal. Le climat de la zone (figure 2) est de type méditerranéen subaride à aride, à hiver froid à tempéré, avec un gradient bioclimatique décroissant du nord au sud. Les précipitations sont faibles et irrégulières et les températures moyennes annuelles avoisinent $19{ }^{\circ} \mathrm{C}$. Les vents sont très fréquents et violents : le Chergui estival peut occasionner de véritables tempêtes de sable remontant du Sahara et contribuer nettement à l'élévation de l'évapotranspiration qui avoisine 1200 mm/an (23).

Les grandes formations végétales rencontrées dans la zone sont les steppes à armoise blanche, les steppes à alfa, les steppes à chénopodiacées et les steppes désertiques présahariennes. Avec le phénomène de la dégradation, ces formations connaissent une dynamique régressive en faveur des steppes dégradées à Noaea mucronata, ou mixtes (Anabasis, asphodèles, Thymelaea et Peganum harmala), et

\section{Tableau II}

Cheptel (têtes) dans la zone d'étude située dans I'Oriental au Maroc en 2010

\begin{tabular}{lrccc} 
& Ovins & Caprins & Bovins & $\begin{array}{c}\text { Densité } \\
\left(\mathbf{U P B} / \mathbf{k m}^{2}\right)\end{array}$ \\
\hline Béni Mathar & 83830 & 12720 & 2500 & 57 \\
Maâtarka & 272210 & 41680 & 1100 & 45
\end{tabular}

UPB : unité de petit bétail (ou équivalent ovin)

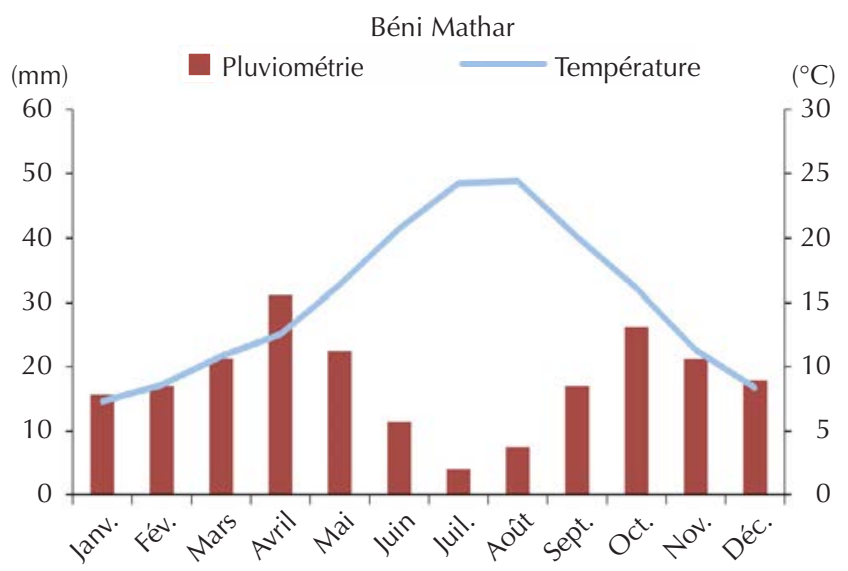

à psammophytes et gypso-halophytes (3). Dans ces différentes formations, une végétation annuelle à cycle court se développe. Les études réalisées sur les parcours de la zone dénotent une évolution régressive du couvert végétal (23) et mettent l'accent sur le phénomène de désertification prononcée dans la zone. D'ailleurs, la productivité végétale rapportée à l'hectare demeure en général très faible et ne dépasse pas 80 unités fourragères (UF)/ha.

\section{Collecte et traitement des données}

L'étude de la dynamique de la végétation s'est appuyée sur une analyse de l'importance des principales formations pastorales en 1970,1988 et 2013. Pour 1970, les cartes d'occupation du sol (15) ont été scannées, puis les formations végétales ont été digitalisées. Les images Landsat des printemps 1988 et 2013 (www.usgs. gov) ont permis d'établir des estimations basées sur les classifications supervisées (sur Arcgis) sur la base des données provenant de l'étude de la cartographie des parcours en 1988 (9), et de nos propres observations et mesures de la végétation réalisées en 2013.

L'étude des causes du changement du système pastoral a été menée selon le modèle forces, pressions, état, impacts, réponses (FPEIR). Ce modèle permet d'analyser les relations entre des facteurs qui ont une incidence sur l'environnement selon une logique de causalité avec des indicateurs classés en fonction de l'étape du cycle de l'approche (25). Les forces représentent les perturbations indirectes relatant la problématique de la zone, et les pressions décrivent les actions ou activités induisant directement les changements.

L'effet de la dégradation sur les systèmes d'élevage et les stratégies d'adaptation a été estimé au travers d'une enquête auprès d'un échantillon de 85 éleveurs (35 à Béni Mathar et 50 à Maâtarka) choisis parmi les listes de vaccination et représentant les différentes tailles des élevages : les petits élevages (moins de 50 têtes), les moyens (50 à 200 têtes) et les grands (plus de 200 têtes).

Le questionnaire structuré utilisé a porté sur la situation des éleveurs et les composantes de l'élevage, en l'occurrence l'utilisation des parcours (choix des sites, règles d'accès, conflits) et la conduite de troupeaux (aspect technique d'élevage, main d'œuvre et relève). L'analyse factorielle a été faite par SPSS sur les variables et les indicateurs clés du questionnaire, ce qui a permis de regrouper ces variables en six facteurs (race, degré d'intensification de l'élevage, superficie possédée, effectif des animaux, paramètres de croissance, et couverture des besoins alimentaires) dont les trois premiers se sont avérés discriminants entre les communes étudiées.

Figure 2 : diagrammes ombrothermiques (1931-2013) pour les deux communes situées dans l'Oriental au Maroc. 
L'analyse de l'évolution des effectifs a été basée sur les informations des services d'élevage (actuellement Office national de sécurité sanitaire des produits alimentaires) et les statistiques disponibles ont permis de considérer le capital de femelles reproductrices pour éviter les divergences rencontrées lorsque les animaux de l'année étaient intégrés et pour procéder aux calculs des charges animales. Les charges réelles (ou effectives) ont été basées sur l'estimation du nombre d'animaux par hectare de parcours, alors que les intensités d'exploitation des pâturages ont été déduites du rapport entre la charge animale réelle et la capacité de charge.

La perception du climat et les stratégies d'adaptation ont été discutées au cours d'ateliers participatifs comprenant de 10 à 25 personnes (élites, éleveurs et personnes concernées par la gestion des parcours). Les débats, orientés par un animateur confirmé, ont porté notamment sur les pâturages, l'élevage, l'évolution de la taille des troupeaux, le mode de gestion. Les informations discutées ont été capitalisées par deux rapporteurs équipés de magnétophones (pour compléter ultérieurement les notes prises).

\section{RESULTATS}

\section{Dégradation des ressources pastorales}

La tendance à la désertification (34) s'est manifestée au niveau de la zone de l'étude depuis au moins les trois dernières décennies par une dynamique régressive des bons sites pastoraux (à alfa et à armoise), voire même des sites en état moyen. Ils ont été remplacés par des espaces défrichés et des formations dégradées où dominent des espèces indicatrices de la dégradation (figure 3 ). Les principales formations pastorales ainsi identifiées sont :
- les steppes à alfa en bon état ; formations où l'alfa (Stipa tenacissima) domine les élévations et les zones protégées ; les espèces en association comprennent Thymus sp., Helianthemum sp., Asphodelus microcarpus, Artemisia herba-alba, Lygeum spartum, Noaea mucronata, Peganum harmala et Atractylis sp.;

- les steppes à alfa moyennement dégradées qui dominent la majeure partie du paysage ; les espèces en association comprennent les annuelles, Schismus barbatus, des asphodèles et Noaea mucronata sur les sols sableux, et Lygeum spartum sur les sols caillouteux et sablo-limoneux ;

- les faciès à alfa très dégradés ; l'alfa a disparu ou est en voie de disparition; les espèces de remplacement sont Noaea mucronata et Lygeum spartum sur les sols caillouteux et sablo-limoneux ;

- les faciès à armoise blanche (Artemisia herba-alba) ; faciès des bas-fonds, des lits d'oueds et zones de dépression ; l'armoise est associée à diverses espèces selon l'état de dégradation des faciès ; dans les faciès en bon état, on rencontre Lygeum spartum, Atractylis serratuloides, Thymelaea microphylla, Astragalus armatus, Stipa parviflora, Schismus barbatus et Plantago albicans; dans les faciès dégradés, on trouve surtout Noaea mucronata, Anabasis aphylla, Atractylis serratuloides et Peganum harmala;

- les formations de dégradation ; il s'agit essentiellement de a) steppes à Noaea mucronata qui remplacent les steppes à alfa sur sols caillouteux, b) faciès à Anabasis qui remplacent les faciès à armoise sur les parcours dégradés et c) steppes à Peganum harmala qui colonisent aussi les faciès à armoise soumis à des pressions de pâturage.

Les mises en culture sont éparpillées dans la zone (phénomène de mitage des parcours) au niveau des dépressions dominées auparavant par l'armoise, au niveau des lits des oueds et des piémonts, et
$\hat{A}$

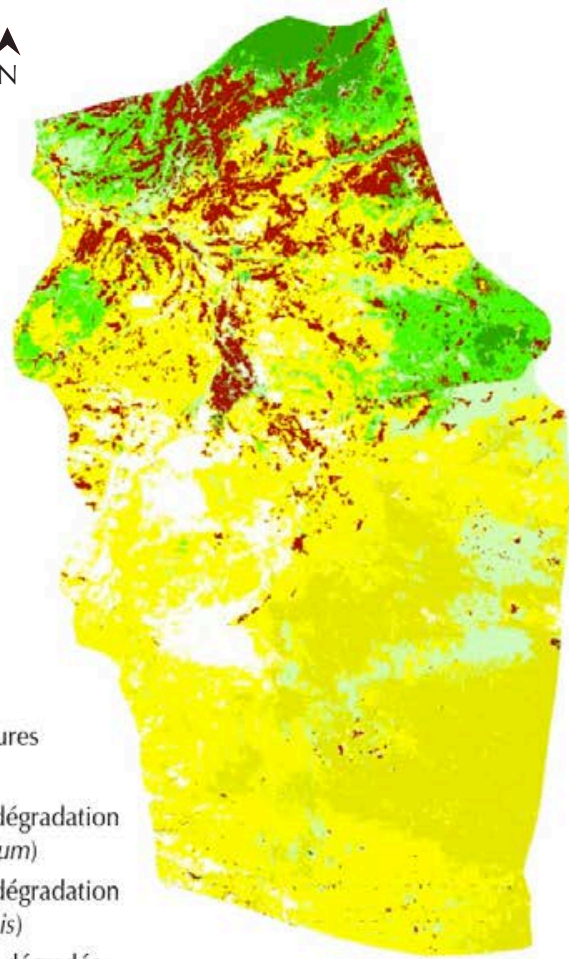

Formation à armoise très dégradée

Formation à alfa très dégradée

Formation à alfa moyenne à très dégradée Formation à alfa dégradée

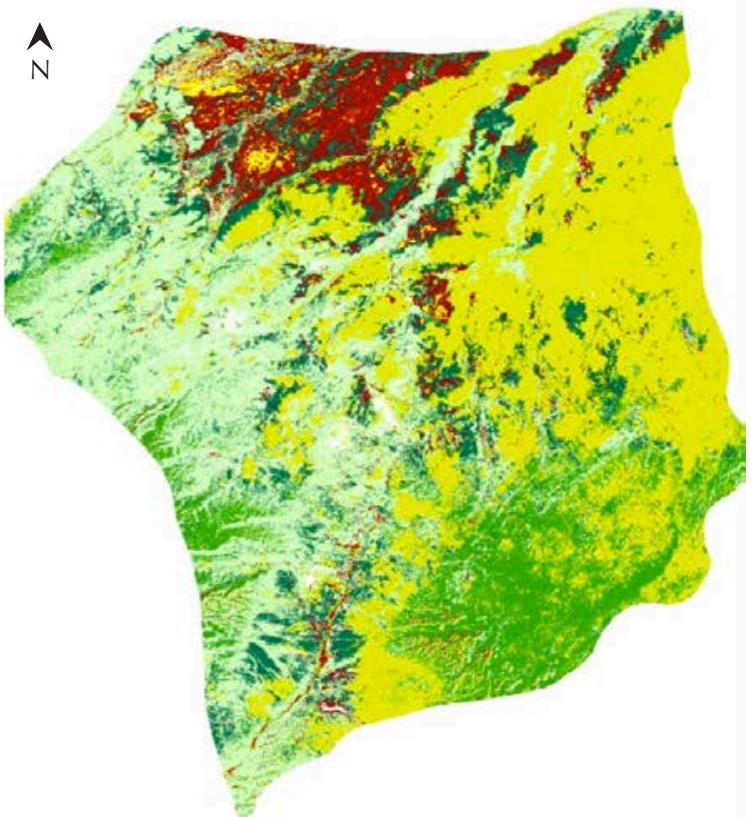

Terrains très défrichés et cultures Sol nu

Formation à Tamarix, Retama (oueds)

Formation à espèces de dégradation (Anabasis, Peganum)

Formation à armoise très dégradée

Formation à alfa très dégradée

Formation à alfa moyenne à dégradée

Figure 3 : cartes d'occupation des sols des communes de l'étude situées dans l'Oriental au Maroc. 


\section{Tableau III}

Evolution des superficies (ha) des principales formations pastorales dans la zone d'étude située dans l'Oriental au Maroc

\begin{tabular}{|c|c|c|c|c|c|c|c|}
\hline & CR * & 1970 & $\%$ & 1988 & $\%$ & 2013 & $\%$ \\
\hline \multirow[t]{2}{*}{ Faciès à alfa en bon état } & 1 & 35074 & 21 & 0 & 0 & 0 & 0 \\
\hline & 2 & 223837 & 32 & 0 & 0 & 0 & 0 \\
\hline \multirow[t]{2}{*}{ Faciès à alfa en moyen état } & 1 & 20548 & 12 & 18538 & 11 & 4892 & 3 \\
\hline & 2 & 33712 & 5 & 94988 & 14 & 80822 & 12 \\
\hline \multirow[t]{2}{*}{ Faciès à alfa dégradé à très dégradé } & 1 & 15096 & 9 & 33027 & 20 & 30800 & 18 \\
\hline & 2 & 55476 & 8 & 105434 & 15 & 225610 & 32 \\
\hline \multirow[t]{2}{*}{ Faciès à armoise moyen à dégradé } & 1 & 65971 & 39 & 24076 & 14 & 0 & 0 \\
\hline & 2 & 196498 & 28 & 308213 & 44 & 0 & 0 \\
\hline \multirow[t]{2}{*}{ Armoise dégradé à très dégradé } & 1 & 2724 & 2 & 32808 & 19 & 29633 & 18 \\
\hline & 2 & 73990 & 11 & 87162 & 13 & 231853 & 33 \\
\hline \multirow[t]{2}{*}{ Terrain dénudé, mise en culture } & 1 & 12639 & 8 & 28038 & 17 & 32962 & 20 \\
\hline & 2 & 3285 & 1 & 3687 & 1 & 12992 & 2 \\
\hline \multirow[t]{2}{*}{ Végétation des oueds } & 1 & 3262 & 2 & 0 & 0 & 0 & 0 \\
\hline & 2 & 63222 & 9 & 48788 & 7 & 88000 & 13 \\
\hline \multirow[t]{2}{*}{ Formations de dégradation } & 1 & 13806 & 8 & 32633 & 19 & 70833 & 42 \\
\hline & 2 & 48102 & 7 & 49842 & 7 & 58837 & 8 \\
\hline
\end{tabular}

*Commune rurale : 1. Béni Mathar ; 2. Maâtarka

là où les possibilités de labours se présentent (possession de terres, récolte de grains ou déprimage).

Les tendances superficielles confirment la première hypothèse puisqu'elles révèlent la disparition des bons faciès à alfa et à armoise (tableau III). Les formations en état moyen ne sont pas à l'abri de la dégradation, particulièrement à Béni Mathar. Les espaces sont davantage occupés par les formations végétales à armoise dégradées à très dégradées, les formations à espèces de dégradation, et par des espaces dénudés ou mis en culture.

Il en résulte des espèces végétales à forte capacité colonisatrice au détriment des formations végétales de base (alfa et armoise blanche) qui abondaient encore au début des années 1970 (tableau IV) (27). Le recouvrement de la végétation, qui reste dans la majorité des cas inférieur à 20 p. 100, est un indicateur clé qui témoigne de la perte de la vigueur de la végétation steppique de la zone.

La dynamique de la végétation régressive s'accompagne aussi d'une baisse remarquable de la phytomasse sur parcours (figure 4). Estimée à environ 29140 tonnes à Béni Mathar et 146680 tonnes à Maâtarka en 1970, la phytomasse a connu une baisse respectivement de 51 et 46 p. 100 en 1988, et de 35 et 27 p. 100 en 2013, soit respectivement environ 60 et 68 p. 100 de baisses enregistrées depuis les années 1970.

\section{Evolution des effectifs et systèmes alimentaires}

Devant la situation critique des ressources pastorales, l'analyse de l'évolution des effectifs révèle une augmentation du cheptel de la

\section{Tableau IV}

Evolution de la flore et du recouvrement des principaux faciès pastoraux dans la zone d'étude située dans I'Oriental au Maroc

\begin{tabular}{|c|c|c|c|}
\hline & Type de faciès & $\begin{array}{c}\mathrm{RGA}^{1} \\
(\%)\end{array}$ & $\begin{array}{c}\mathrm{PF}^{2} \\
\text { (UF/ha) }\end{array}$ \\
\hline \multirow[t]{2}{*}{ 1970-71 } & Faciès à alfa & $15-35$ & 200 \\
\hline & Faciès à alfa et à armoise & $10-25$ & 250 \\
\hline \multirow[t]{3}{*}{ 1989-90 } & Faciès à alfa & $12-24$ & 50 \\
\hline & Faciès à alfa et à armoise & $4-16$ & 80 \\
\hline & Faciès à Noaea mucronata & $9-16$ & 25 \\
\hline \multirow[t]{3}{*}{ 2003-04 } & Faciès à alfa dégradé à très dégradé & $3-15$ & 35 \\
\hline & Faciès à Peganum harmala & $10-20$ & 35 \\
\hline & Faciès à Noaea mucronata & $<8$ & 5 \\
\hline
\end{tabular}

${ }^{1}$ Recouvrement global aérien des espèces pérennes

${ }^{2}$ Production fourragère des faciès dominants

zone qui aggrave davantage les possibilités de couvrir les besoins croissants des animaux sur parcours (figure 5). On observe, depuis le début des années 1990 jusqu'à l'an 2000, un accroissement annuel moyen des ovins de 6,2 et 5,2 p. 100 respectivement à Béni Mathar et Maâtarka, suivi d'une baisse respective de 


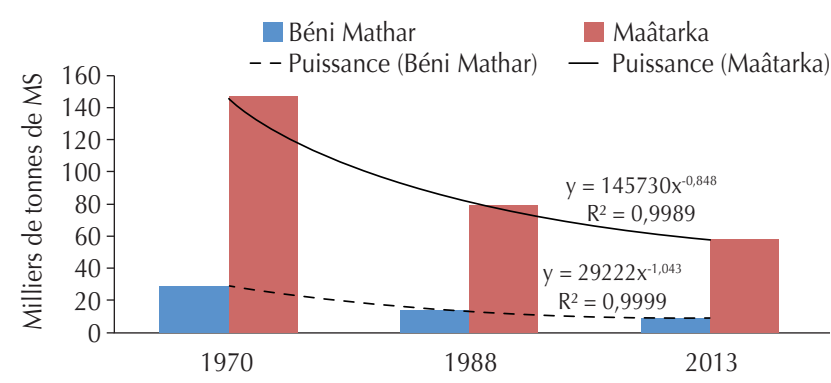

Figure 4 : comparaison de la phytomasse des parcours entre les années 1970 (ERES, 1970) et 1980 (Berkat et Hammoumi, 1990) pour les deux communes de l'étude situées dans l'Oriental au Maroc. Poly. : polynomiale. MS : matière sèche.

1 et 1,5 p. 100 après 2004. En revanche, le nombre de caprins a augmenté en moyenne respectivement de 7,8 et 8,9 p. 100. Ceci montre clairement que la dégradation des pâturages n'a pas été suivie d'une baisse des effectifs d'ovins, de sorte que la dégradation ne peut que s'accentuer.

Les charges réelles, estimées par le nombre d'animaux à l'hectare, sont nettement élevées par rapport à l'optimum toléré par les pâturages de la zone, soit 0,25 unité de petit bétail (UPB)/ha (11). Elles montrent des valeurs plus élevées à Béni Mathar, zone nord, qu'à Maâtarka, zone sud. Les tendances affichent une augmentation continue au nord et une certaine stabilité au sud.

Les capacités de charge, effectifs maximaux de bétail par unité de surface, que les pâturages peuvent supporter sans se détériorer affichent au cours des deux derniers siècles des baisses linéaires $\left(\mathrm{y}=-0,001 \mathrm{x}+\right.$ constante, $\left.\mathrm{r}^{2}=0,99\right)$, avec des moyennes respectives de 0,10 et 0,12 tête par hectare à Béni Mathar et Maâtarka (calculs basés sur les effectifs et des estimations approchées de productions des parcours).

Les intensités d'exploitation des pâturages, rapport entre la charge animale réelle et la capacité de charge, affichent des moyennes de 4,97 à Béni Mathar $(\sigma=2,11)$ et de 3,32 à Maâtarka $(\sigma=0,77)$. Toutes les valeurs affichées dépassent 1 (signe de surexploitation des pâturages) et deviennent plus fortes avec le temps, surtout à Béni Mathar.

La dégradation des ressources, en quantité et en qualité, provoque une baisse importante de l'offre fourragère sur parcours. Cette offre demeure nettement faible par rapport aux besoins des animaux et s'amplifie avec le temps (figure 6).

La tendance linéaire des bilans alimentaires calculés depuis le début des années $1990\left(\mathrm{y}=-80525 \mathrm{x}+2 \mathrm{E}+09 ; \mathrm{R}^{2}=0,9\right)$ a permis de simuler les situations des années passées. Les résultats suggèrent que le point de décrochement de l'écosystème pâturé (point où les parcours ne peuvent plus subvenir aux besoins alimentaires des animaux) pourrait avoir eu lieu au début des années 1940. Suite à ce déséquilibre, les éleveurs ont eu recours à des aliments de supplémentation riches en concentrés (tableau $\mathrm{V}$ ) pour répondre aux besoins croissants du cheptel.

La majorité des éleveurs de Béni Mathar se caractérise par le recours aux parcours (les grands mouvements du cheptel sont quasi abandonnés) et fréquemment à la supplémentation (de 0,5 à $1 \mathrm{~kg} / \mathrm{tête} /$ jour) qui couvre environ 40 p. 100 des besoins des troupeaux dont la taille est très moyenne (90 UPB). L'orge et le son, introduits des zones extérieures, sont les aliments les plus utilisés. L'utilisation de pulpe sèche de betterave (PSB), de paille et de luzerne (généralement disponible sur le périmètre irrigué de Béni Mathar) vient ensuite, avec une baisse entre mai et septembre
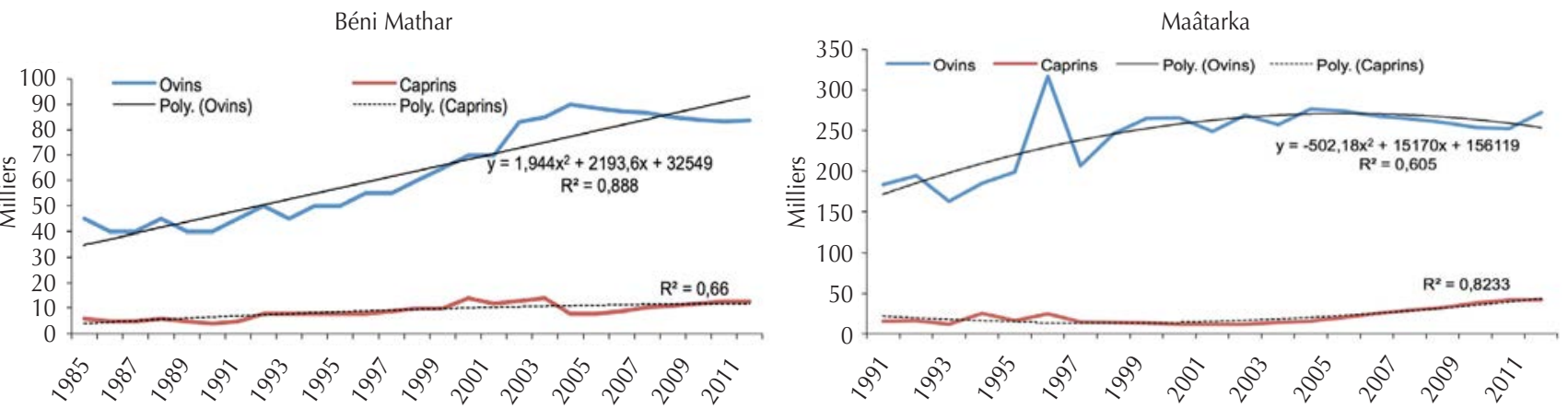

Figure 5 : évolution des effectifs des animaux pour les deux communes situées dans l'Oriental au Maroc. Poly. : polynomiale.
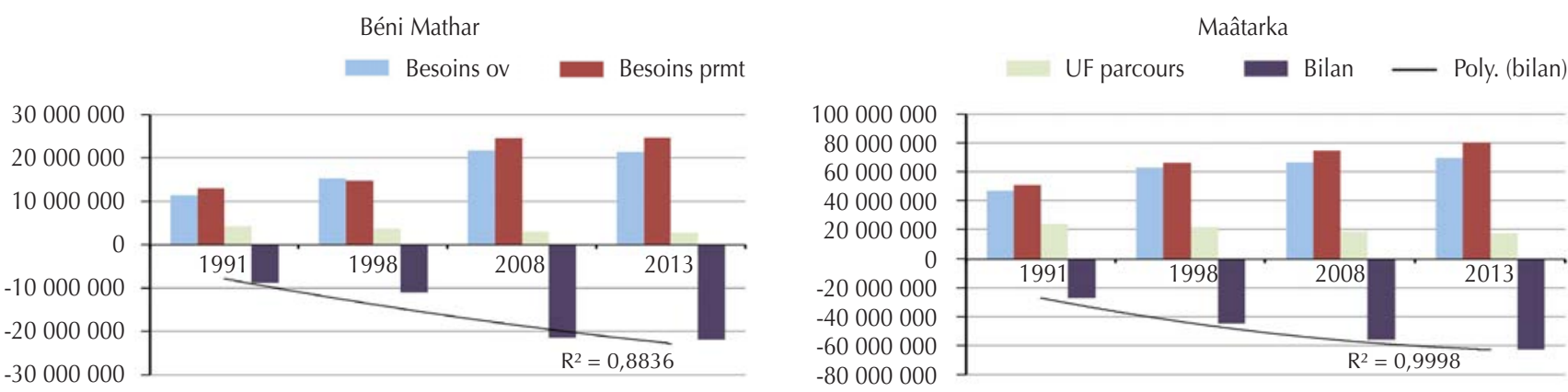

Figure 6 : confrontation des besoins du cheptel aux apports du parcours pour les deux communes de l'étude situées dans l'Oriental au Maroc. Ov : ovin ; prmt : petits ruminants ; poly. (bilan) : corrélation polynomiale pour le bilan entre besoins et apports ; UF : unité fourragère. 


\section{Tableau V}

Quantités moyennes d'aliments utilisés (quintaux/élevage/an) dans la zone d'étude située dans I'Oriental au Maroc

\begin{tabular}{lcc} 
& Béni Mathar & Maâtarka \\
\hline Unité fourragère / an & 18049 & 28786 \\
Orge grain & 94 & 181,5 \\
Son & 73 & 117 \\
Luzerne & 34,5 & 1,3 \\
Mais & 4,3 & 21 \\
Pulpe sèche de betterave & 24 & 8 \\
Paille & 16 & 53,5 \\
Aliment composé & 1,5 & 3,4
\end{tabular}

(lorsque les chaumes sont disponibles). L'avoine est faiblement employée.

Les élevages de Maâtarka sont de type naisseur, basés sur la mobilité des troupeaux d'assez grande taille (320 UPB), avec 30 p. 100 des éleveurs pratiquant des déplacements n'excédant pas 30 kilomètres et 14 p. 100 pratiquant des mouvements de grande amplitude. La supplémentation couvre environ 20 p. 100 des besoins et repose essentiellement sur l'orge et le son (aliments les plus disponibles dans les souks locaux) distribués entre octobre et avril, et en période de disette.

\section{Stratégies d'adaptation}

Les données collectées auprès des éleveurs des différentes tailles de troupeaux (tableau VI) reflètent des comportements variables face aux effets de la sécheresse, qui dépendent nettement du statut socio-économique de l'éleveur.

Tout d'abord, les petits éleveurs, avec en moyenne 27 têtes de femelles reproductrices $(\sigma=10,6)$, de type élevage de subsistance, ont un comportement passif face aux effets de la sécheresse car ils manquent d'anticipation lors d'années de sécheresse. Ils rencontrent fréquemment des problèmes financiers pour subvenir aux différents besoins de leurs familles et des troupeaux et se plaignent souvent des grands éleveurs qui exploitent davantage l'espace (les mises en repos, l'appropriation de grands espaces de pâturages et de cultures) et profitent mieux des redevances et subventions. L'effectif du troupeau varie en raison de la vente fréquente des animaux. Une majeure partie de ce groupe est continuellement à la recherche d'activités annexes liées ou non à l'élevage (en

\section{Tableau VI}

Stratification socio-économiques des éleveurs et stratégies dans la zone d'étude située dans l'Oriental au Maroc

\begin{tabular}{lcccc} 
& Nb. & $\begin{array}{c}\text { Taille du } \\
\text { troupeau }\end{array}$ & $\begin{array}{c}\text { Importance } \\
(\%)\end{array}$ & $\begin{array}{c}\text { Cheptel } \\
(\%)\end{array}$ \\
\hline Grands éleveurs & 12 & Plus de 200 têtes & 14 & 65 \\
Eleveurs moyens & 15 & 50 à 200 têtes & 18 & 19 \\
Petits éleveurs & 58 & Moins de 50 têtes & 68 & 16
\end{tabular}

l'occurrence le gardiennage des troupeaux des plus grands éleveurs). L'abandon de l'élevage, l'exode et l'émigration sont des scénarios souvent envisagés par cette catégorie possédant des troupeaux de faibles effectifs, entretenus par la main-d'œuvre familiale (gardiennage, alimentation), avec moins de deux ouvriers occasionnels par élevage et par an pour assurer la tonte.

Ensuite, les exploitations pastorales intermédiaires, avec en moyenne 96 brebis $(\sigma=38,8)$, sont plus à l'aise que les petits éleveurs et trouvent des intérêts mutuels avec les deux autres catégories. Pour elles, le troupeau est un moyen pour survivre et n'est pas, dans l'ensemble, conçu comme un capital à rentabiliser. Généralement, cette catégorie combine l'opportunité de déplacer les troupeaux vers des sites moins dégradés pour minimiser les coûts de la supplémentation, la vente d'animaux et l'approvisionnement régulier en suppléments. La taille de l'élevage fluctue souvent puisqu'il est la source principale de revenus. La main d'œuvre familiale est utilisée plus fréquemment que celle rémunérée, avec deux ouvriers occasionnels en moyenne par exploitation par an pour s'occuper de la tonte et un berger permanent.

Enfin, les exploitations les plus grandes, avec en moyenne 415 brebis $(\sigma=199)$, sont caractérisées par un niveau de gestion plus concret et visent la réalisation de profits et la rentabilisation du capital (Acherkouk et coll. les qualifient de spéculateurs [2]), notamment dans des activités liées à l'élevage et probablement dans d'autres secteurs ou activités (non déclarées). Cette stratégie se manifeste par la possession d'un effectif d'animaux plus important, l'appropriation de grandes superficies de terres de parcours et l'acquisition d'équipement (camion, tracteur, entre autres). Pour surmonter les effets de la sécheresse, la mobilisation des fonds de l'épargne réalisée en période favorable est la solution pour entretenir un grand effectif d'animaux reproducteurs et engraisser les jeunes destinés à la vente, avec des stocks d'aliments achetés bien avant la baisse des ressources alimentaires sur parcours et la possibilité de transhumer ailleurs (plus rare au nord qu'au sud). Cette stratégie permet à cette catégorie de surmonter plus facilement les contraintes et ainsi de garder les animaux au lieu de les vendre à bas prix. L'utilisation de la main d'œuvre salariée est fréquente pour réaliser la plupart des tâches liées à l'élevage et à la commercialisation.

L'âge moyen des éleveurs était assez avancé ; plus de 50 p. 100 des éleveurs avaient plus de 55 ans. Avec un taux d'analphabétisme élevé (85 p. 100), l'avenir de l'élevage dans la zone semble incertain surtout chez les éleveurs les moins disposés à adopter de nouvelles technologies (2) et parce que les plus jeunes éleveurs rêvent d'émigration.

Les ventes demeurent une politique généralisée à caractère spéculatif chez les grands éleveurs qui vendent à la bonne période et valorisent mieux leur engraissement. Elles sont sécurisantes pour les plus petits qui ne vendent pas toujours au meilleur moment. Elles concernent presque toutes les catégories d'animaux. Les plus vendus sont les jeunes de six à douze mois (40 p. 100 des ventes totales réalisées), suivis des plus jeunes de moins de six mois (36 p. 100) et des plus âgés de plus d'un an (24 p. 100).

\section{DISCUSSION}

Ce travail a été confronté à des difficultés liées à la disponibilité des informations antérieures et à leur accès. Il a été nécessaire de contacter des personnes clés pour rassembler des études anciennes et les compiler. Des biais potentiels restent toutefois possibles en raison d'approches et de techniques de suivis différentes pour 
l'étude de la végétation, de la fiabilité des statistiques exploitées et de la typologie d'éleveurs utilisée.

L'histoire de la zone met en évidence le fait que la société pastorale est dotée d'une forte capacité d'adaptation dictée par l'importance de l'activité d'élevage, liée aux vastes espaces des parcours. Cependant, les perturbations vécues (socio-économique, politique et environnementale) ont modifié les modes de vie des pasteurs qui recherchent de plus en plus les services urbains, le bien-être et des activités à revenu plus stables que celui de l'élevage pastoral, très influencé par les aléas.

En effet, la production des parcours de la zone est très affectée par la dégradation des bons faciès à alfa et à armoise alors que ces derniers abondaient encore au début des années 1970. Les formations pastorales en état moyen ne sont pas non plus à l'abri de la dégradation. Les espaces sont de plus en plus occupés par les formations végétales à armoise dégradées à très dégradées et par des espaces dénudés ou mis en culture. La situation est devenue plus alarmante avec l'évolution négative et exponentielle de l'offre fourragère des pâturages, davantage occupés par des espèces de plantes plus ou moins appétibles (23) et leur forte capacité colonisatrice.

La dégradation des parcours, estimée par la régression des ressources pastorales et la diminution de la biomasse des espèces pérennes (4), est constatée dans la zone depuis plus de quatre décennies. Les actions de restauration réalisées, en particulier par les projets de développement des parcours et de l'élevage dans la zone, ne semblent pas stopper ce processus malgré la multitude et la diversité de ces actions (notamment mise en repos, plantations, organisation des usagers en coopératives pastorales et unions, redevance sur le pacage, études sur la stratégie des parcours et usages des ressources). Les difficultés liées à la sécheresse et au respect des actions d'aménagement par les usagers sont les plus notées bien que des mesures de contrôle (gardiennage) et de sanction (police pastorale) avaient été fermement prises. Pour homogénéiser le langage des départements ministériels concernés (d'autant que l'organe de tutelle des terres collectives, le ministère de l'Intérieur, privilégie la sûreté publique), un accord tripartite entre les ministères de l'Agriculture, des Eaux et Forêts et de l'Intérieur a été adopté (on y reproche l'absence des usagers et le retard dans la mise en œuvre).

Outre les limites techniques des organes de gestion étatiques et les contraintes de la diversification des espèces végétales utilisées (principalement Atriplex nummularia), la responsabilisation de la population dans la restauration des sites dégradés manque considérablement malgré la constitution de coopératives pastorales. En effet, même si les éleveurs s'accordent sur le fait que le paysage végétal dans la région est prédominé par des espèces à très faible qualité pastorale et que dans la majorité des couverts les végétaux sont très peu appétibles ou ne le sont pas, rares sont ceux qui reconnaissent leur responsabilité dans cette dégradation (surpâturage, défrichement pour le bois-énergie, labour des terres de parcours, utilisation irrationnelle des parcours). Ils préfèrent accuser les épisodes de sécheresse. L'impact de la sécheresse est, en fait, faible ou négligeable là où l'impact humain et animal est faible ou nul (22). La végétation et les sols des zones arides se sont adaptés à des conditions de sécheresse récurrentes au cours des siècles passés (22).

L'analyse climatique réalisée sur une série de données collectées depuis les années 1930 (figure 7) montre une tendance à la baisse des précipitations, comme observé par Mahyou et coll. (23). Ces derniers relèvent une fréquence croissante des années de sécheresse, passant pendant les trois dernières décennies de deux à trois années sur cinq (23). La fréquence élevée des années de sécheresse et la baisse de la moyenne pluviométrique annuelle ont affaibli la capacité de régénération des espèces végétales et fragilisent les systèmes de production (33). Le facteur anthropique reste le plus pesant dans la dynamique défavorable que connaît la zone. Les contraintes physiques et climatiques ne constituent dans la plupart des cas que des facteurs favorables (31).

Les pasteurs ont acquis un savoir sur la dynamique des écosystèmes qui les amène à déceler les changements et à s'adapter en conséquence. Ils avouent être moins capables que par le passé (surtout avant les années 1960) de supporter les effets de la sécheresse, et ce, en raison de la baisse de la mobilité des troupeaux qui permettait d'exploiter des pâturages plus favorables. On notait à l'époque le respect des règles et des usages coutumiers tribaux, et une certaine concordance entre les effectifs élevés et l'offre de parcours. Pour certains, les petits éleveurs sont les plus vulnérables au changement climatique (7), pour d'autres, ce sont les grands éleveurs qui en souffrent le plus puisqu'ils utilisent davantage les ressources naturelles (30). Avec des moyens moins limités, ces derniers arrivent à exploiter plus d'espace sans pour autant éviter le devoir de respecter les droits de pacage des autres.

Paradoxalement à la sédentarisation cadencée des troupeaux, on assiste à l'évolution des effectifs des animaux (figure 5). La culture ancestrale et le positionnement social ont toujours marqué

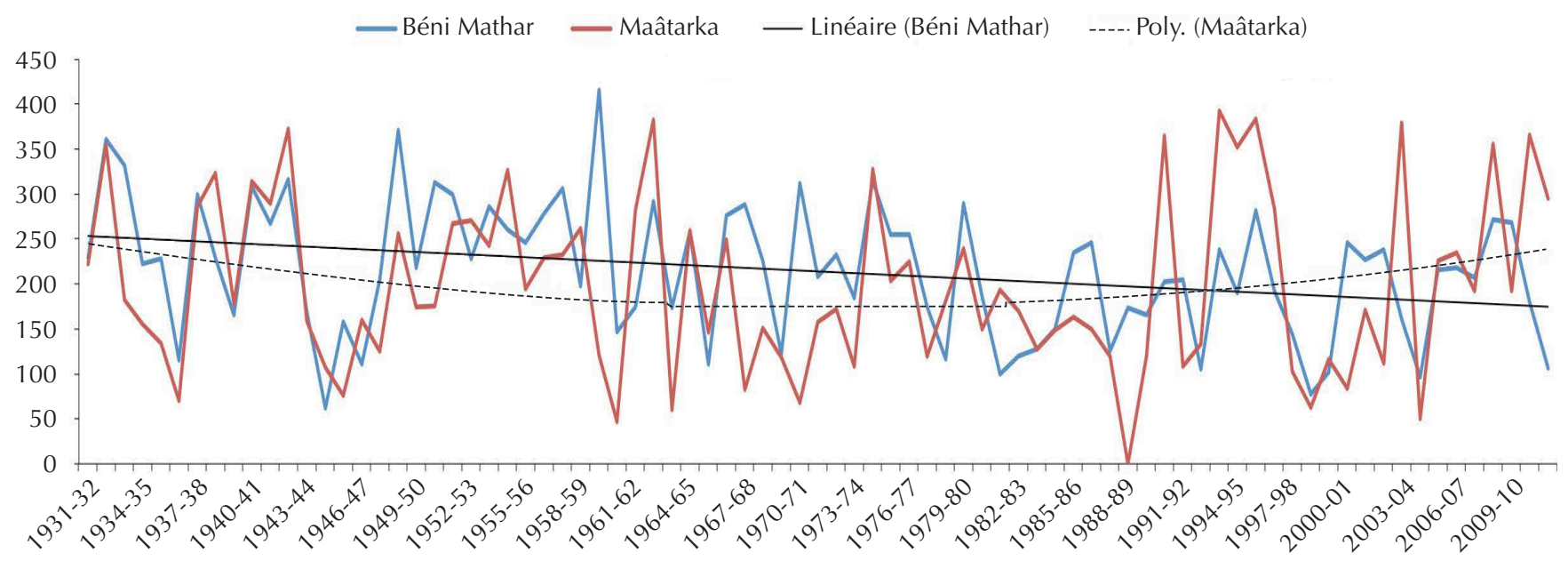

Figure 7 : pluviométries moyennes annuelles (Maâtarka relève de la station de Tendrara) pour les deux communes de l'étude situées dans l'Oriental au Maroc. Poly. : polynomiale. 
les éleveurs avec davantage d'animaux. Mais l'augmentation des effectifs constatée ne peut se passer sans pression nuisible sur les ressources pastorales, imposant impérativement la supplémentation des troupeaux dont les besoins n'ont pas été satisfaits sur parcours (les enclos d'élevage, ou zribas, sont transformés en bergeries à ciel ouvert).

L'élevage est considéré rentable et regardé comme une activité noble au sein de la société rurale. Le maintien des animaux de moins en moins mobiles et en nombre bien supérieur aux capacités de charge des parcours (28) s'explique par le recours à la supplémentation des animaux qui profitent aussi des actions encourageantes des programmes de développement (mises en repos, points d'eau, subventions d'alimentation, interventions sanitaires, etc.).

La concentration des animaux, émanant de comportements opportunistes des individus sans règles collectives de bonne gestion, est notée sur des sites particuliers et autour des points d'eau avec des itinéraires de pâturage plus rétrécis et des temps de séjour plus longs, sans prise en compte de la période active de la végétation. Ces attitudes, individuelles ou collectives, se mettent en place, pour certains éleveurs, par la sélection et l'appropriation des meilleurs parcours (corrélées au niveau de la richesse moyenne des douars [groupement d'habitations] ou résultat d'un rapport de force historique entre les fractions [entités sociales formant les tribus]) et, pour d'autres, par les mises en culture (corrélées à la structure démographique des fractions) permettant ainsi d'accéder à de vastes espaces pastoraux (12). La dominance par la mise en culture n'a aucun impact sur les décisions individuelles, alors que celle par le pâturage réduit les risques et les vulnérabilités (12).

La prolifération des moyens de transport a été fortement influencée par la multiplication du nombre de véhicules ou engins (essentiellement le camion qui a remplacé le dromadaire) qui traversent le pâturage en toute saison et favorisent la montée en puissance des grands troupeaux (33). Ces troupeaux de 300 têtes (au nord) et 600 têtes (au sud), gardés par un berger expérimenté (avec un contrat informel annuel très précis), exploitent plus d'espace et deviennent une cause majeure de dégradation (forte pression) lorsque les séjours sont prolongés sur le même site ou si plusieurs troupeaux se succèdent. En effet, les grands troupeaux ne peuvent passer sur les ressources sans incidence. Benbrahim et coll. (8) notent que la dégradation dans la zone aride est corrélée avec une charge animale excessive. En maintenant des effectifs de plus en plus grands, de moins en moins mobiles et bien supérieurs à la capacité de charge (33), la pression sur les pâturages est considérée comme étant l'une des principales causes anthropiques de la dégradation des parcours de la zone. Selon Tag (32) le surpâturage n'est pas nouveau, il est antérieur à la sécheresse de la fin des années 1970 début 1980

La passivité des pouvoirs publics et de la société civile vis-à-vis de l'installation du processus de désertification a permis aux éleveurs d'accaparer de grands espaces steppiques et de les mettre à nu, par la mise en culture et le surpâturage (33). La mobilité qui assurait les besoins des animaux toute l'année par l'exploitation de différents sites est devenue, depuis les années 1960, seulement une alternative car une majorité d'éleveurs déclare avoir cessé les déplacements après la sécheresse des années 1970 (29).

L'abandon du dromadaire, progressivement remplacé par le camion qui a rendu le système nomade sélectif (29), peut être considéré comme une rupture marquante du système pastoral d'autrefois. Mais le recul de la transhumance trouve des explications dans les contraintes climatiques, sociopolitiques et administratives, donnant lieu à une sédentarisation généralisée qui caractérise la crise des pasteurs, développée depuis le début du $\mathrm{XX}^{\mathrm{e}}$ siècle (32). Selon certains auteurs $(21,32)$, la mobilité pastorale était jugée par les administrateurs coloniaux (voire postcoloniaux) comme incompatible avec le progrès technique et le développement socio-économique, et aucune innovation ne pouvait être introduite en milieu pastoral tant que subsisterait le mode jugé archaïque de production de l'élevage transhumant.

Aujourd'hui, rares sont les éleveurs qui cherchent à exploiter des parcours hors zones ou à réduire les effectifs pour s'adapter à l'offre fourragère disponible. La disponibilité des moyens de transport et de stockage d'eau et d'aliments a facilité la fixation des troupeaux et leur maintien sur des sites dégradés. «L'eau et les concentrés viennent maintenant vers les troupeaux» (33).

Les coûts de production au niveau de la zone, incluant principalement les frais d'alimentation, d'amortissement et de main d'œuvre, sont estimés entre 62000 et 687000 dirhams (dh) par an (soit 5710 et $63280 € /$ an), et les marges bénéficiaires nettes réalisées sont entre 19000 et $550300 \mathrm{dh}$ (1 750 et $50690 €)$ selon la taille de l'élevage (2). La supplémentation, cette charge lourde qualifiée de burlesque par les éleveurs de la zone (29), surtout avant les années 1970 car pour profiter des ressources il suffisait d'allonger le temps de séjour des bêtes, a été adoptée progressivement, particulièrement au début des années 1980. L'introduction de quelques aliments sert d'ailleurs de repère historique, comme par exemple celle de la pulpe sèche de betterave en 1978-79 et de l'aliment composé en 1989. Elle a été favorisée par un marché actif d'aliments de bétail et par les actions encourageantes de l'Etat (programme de sauvegarde du cheptel, subventions pour les aliments et le transport, essais d'alimentation menés chez les éleveurs par les directions provinciales de l'agriculture et par les centres de travaux agricoles, etc.), particulièrement avec la mise en place du projet de développement des parcours et de l'élevage de l'Oriental. Les pouvoirs publics ont largement contribué à l'essor de la pratique de la supplémentation (10), suite à la prise de conscience que le sort des éleveurs relevait de leur responsabilité.

En fait, les animaux n'arrivent pas à satisfaire leurs besoins quelles que soient les conditions météorologiques de l'année. Le Fonds international de développement agricole (FIDA) (18) rapporte que les apports sont estimés à 57 millions, 113 millions et 170 millions d'UF pour les besoins des animaux respectivement de 188 millions d'UF en année sèche, 214 millions d'UF en année moyenne et 226 millions d'UF en année bonne.

Les rations des compléments sont composées essentiellement d'orge grain (57 à 66 p. 100), de son (18 à 28 p. 100) et de l'aliment composé $(3,4$ à 9,5 p. 100). Les apports sont utilisés pour supplémenter les animaux, surtout en période de disette, en privilégiant les animaux sensibles (fin de gravidité, début de lactation) ou destinés à la vente. L'engraissement des jeunes est considéré bénéfique pour la décongestion des parcours (19) et plus rémunérateur sur les marchés locaux, surtout au moment des fortes demandes de l'année (fête du sacrifice, cérémonies d'été).

Les éleveurs affirment qu'une bonne année pluvieuse est une opportunité à saisir pour minimiser les coûts élevés de la supplémentation. Lors des années bonnes ou moyennes les bons pâturages sont exploités et les effectifs des troupeaux sont augmentés. Malonine, cité par Ouedraogo (26), rappelle que les petits ruminants constituent un disponible monétaire accessible à tout moment et que l'accroissement des effectifs serait une réponse à la monétarisation progressive de l'élevage. Le cas échéant, la supplémentation des troupeaux est le remède incontournable avant de procéder à la vente des animaux. Hormis les effets sociaux discutables (les subventions profitent généralement aux éleveurs les plus aisés) et les retombées économiques globales incertaines, les subventions fourragères en période de sécheresse ont aussi des effets préjudiciables sur les terres de pâturage (16). 
Parallèlement à cette intensification alimentaire, on note un changement dans la composition raciale des troupeaux. Alors qu'ils étaient dominés par la race locale Béni Guil (la région de l'Oriental est officiellement reconnue comme étant le berceau de cette race par arrêté du ministère de l'Agriculture $n^{\circ} 1064$ du 9 novembre 1984), les troupeaux sont de plus en plus envahis par la race blanche algérienne Ouled Djellal. Bon nombre d'interviewés attribuent l'expansion de cette la race à la sédentarisation et à l'intensification des élevages de la zone. Ils pensent que cette race d'origine algérienne aurait des avantages liés à sa précocité, ses performances pondérales et son phénotype plus distingué, lui conférant une bonne valeur marchande et bouchère. La dévaluation du dinar algérien avait amplement influencé les flux incontrôlés entre les frontières et l'expansion de cette race qui représente environ 30 à 50 p. 100 des effectifs de la zone d'étude (2).

Curieusement, l'introduction de cette race dans le centre-ouest de la Tunisie a été jugée comme une cause du quasi-abandon de l'usage de l'espace pastoral du fait de ses besoins élevés (5). La race locale Béni Guil, malgré ses partisans qui privilégient son adaptation et sa rusticité (6), serait plus délaissée sans les efforts de sélection génétique soutenus par les groupements d'éleveurs au sein de l'Association nationale ovine et caprine (ANOC).

L'intensification de l'élevage chez les éleveurs mieux adaptés prend place progressivement lorsque d'autres éleveurs souffrent beaucoup de la sévérité des conditions du milieu qui a obligé un bon nombre d'entre eux à abandonner l'élevage extensif (estimé par les interviewés à 70 p. 100 de la population depuis les années 1970), voire à abandonner définitivement l'élevage (31) et à se tourner vers le travail occasionnel qui produit un revenu plus stable.

La capacité d'adaptation des éleveurs est, certes, dépendante de l'amplitude des variations de la pluviosité vécue, mais la capacité d'anticiper dépend nettement de la taille de l'élevage et des moyens matériels et financiers disponibles. Alors que l'on distingue dans les années 1980 neuf classes d'éleveurs allant des microéleveurs sédentaires aux grands pasteurs transhumants (27), les classes ou types d'éleveurs sont actuellement limités aux petits, moyens et grands sans parfois de distinctions claires. Le passage d'une classe à une autre est souvent observé dans la zone. La pluviosité influence nettement la taille des élevages et détermine par conséquent le passage d'une catégorie à une autre. Les élevages de moins de 70 têtes à Béni Mathar ou de 100 têtes à Maâtarka sont davantage menacés. Les éleveurs de moins de 20 têtes sont considérés pratiquement sans troupeau et doivent se recycler dans d'autres champs d'activités pour survivre (28). Les éleveurs plus aisés ne se sentent pas à l'abri des effets de la sécheresse, surtout s'ils manquent de moyens de financement.

Aggravée par les effets de la sédentarisation généralisée et le maintien des effectifs par la supplémentation, la dégradation des parcours ne peut être stoppée sans cadre stratégique pour le développement durable de la zone intégrant un plan pour le développement des parcours et de l'élevage. Les aménagements pastoraux en zone aride, jugés problématiques (17) dans ces milieux complexes, peuvent suivre le modèle technique et écologique à trois démarches proposé par Aronson, cité par Ferchichi (17), à savoir la restauration (milieux moins perturbés), la réaffectation (remplacer un espace ou un écosystème par un autre de même usage mais de composition floristique différente) et la réhabilitation, mettre un écosystème simplifié inspiré de l'écosystème de référence.

Le plan de développement ou d'aménagement, à caractère interactif et participatif, doit arrêter les choix stratégiques, les textes juridiques, les approches et les programmes d'aménagement et de gestion. Les insuffisances notées dans la nouvelle stratégie marocaine de développement agricole (Plan Maroc Vert, 2009-2020) peuvent être couvertes en adoptant par exemple un plan de gestion écosystémique (11). Mais la centralisation des prises de décision et l'insuffisance des mécanismes institutionnels de concertation entre les acteurs du développement rural (pluralité d'acteurs aux compétences mal réparties et aux responsabilités mal définies) rendent la tâche difficile et affaiblissent davantage les organisations communautaires censées être chargées de la gestion des ressources. Plusieurs rapports (notamment FIDA, BIRD, Icarda) proposent le retour aux institutions locales et encouragent les associations de pasteurs.

La lutte contre la désertification, la dégradation des ressources pastorales et l'atténuation des effets de la sécheresse sont des actions à mener conjointement aux actions de renforcement des connaissances et de développement des systèmes d'observation des ressources naturelles. La lutte contre les pratiques préjudiciables aux ressources naturelles doit être menée en intériorisant les bases de l'approche participative, éléments déterminants (19), vers l'autonomisation des ruraux (24). La mise en culture, souvent non rentable (mois de cinq quintaux par hectare) et visant essentiellement l'appropriation des terres, est à délimiter (7) pour au moins stopper l'extension, comme cela a été décidé par les habitants d'Ouled Sidi Abdelhakem, commune à l'est de Béni Mathar. L'anticipation et le développement de techniques d'adaptation prématurées minimiseront les coûts et permettront de surmonter les effets indésirables. Les travaux de conservation des eaux et des sols sont à prévoir continuellement pour limiter l'érosion, favoriser l'infiltration et protéger la zone contre l'ensablement (19).

Il est important de promouvoir les techniques et les pratiques profitables et respectueuses de l'environnement en assurant l'équilibre entre les prélèvements du cheptel et les capacités de production fourragère des parcours (33). A l'utilisation rationnelle des ressources sous forme de schéma directeur (19) ou système de support de décision (20), à la régulation des charges animales (20) et à la promotion de la mobilité des troupeaux (vers une charge pastorale intertribale basée sur le bon voisinage et la conservation des parcours), s'ajoute l'importance de réhabiliter les espaces dégradés et de reconvertir les espaces défrichés ou dénudés. Il est temps de passer à l'organisation de l'espace pastoral en unités qui tiennent compte de l'organisation sociale (7) et des expériences réussies d'alliance pastorale.

Le recours au pacage raisonné ou rotatif, qui impliquera en premier les usagers potentiels, est vivement conseillé même au plus bas des échelles des communautés (à la recherche d'affinités ethniques). Les actions de restauration combinées à la gestion de charges optimales en période d'ouverture doivent cibler l'amélioration du couvert végétal et la biodiversité (1). La compétition observée entre les coopératives pastorales sur les niveaux de réussite des mises en repos réalisées dans la zone est un acquis qu'il faut retravailler en insistant sur la solidarité sociale, l'appui incitatif et les encouragements (à prévoir dans un fonds de développement pastoral à l'image du fonds de développement agricole). Les actions de reboisement, de plantation et/ou d'ensemencement doivent privilégier la biodiversité en espèces pastorales autochtones. Le problème de la disponibilité des semences est à résoudre par l'installation de réserves semencières dans les zones potentielles et par l'appui aux programmes des centres de recherche et de production (cas du Centre national de production des semences pastorales d'El Jadida et ses antennes régionales).

Si le rôle de l'Etat reste déterminant dans ces écosystèmes fragiles et si du temps est nécessaire au redressement de la situation (26), la réussite de ce plan de développement des parcours et de l'élevage dans la zone repose sur le niveau d'adhésion des populations 
(organisation effective de toutes les catégories et responsabilisation plus concrète des usagers), sur l'intégration du savoir local, sur les réformes institutionnelles (20) et sur le respect des règles et conditions de restauration.

La privatisation, solution préconisée pour certains sites (26), a été nettement déclarée pour l'ensemble de la zone de l'étude par un bon nombre d'usagers (particulièrement à l'atelier de Béni Mathar) et par des chercheurs (dans un atelier organisé à Oujda par l'Icarda en 2001) à l'image du système de ranching. La privatisation au niveau tribal est avancée (14) comme solution alternative au dilemme encore persistant dans les pays du WANA (ouest de l'Asie et nord de l'Afrique) qui considèrent les parcours comme propriété de l'Etat (14).

La durabilité du système ne peut avoir lieu avec l'augmentation des effectifs qui conduit au surpâturage (26). Des considérations telles que la saisonnalité et l'hétérogénéité spatiale sont importantes pour une gestion rationnelle (20). Des décisions législatives et réglementaires en matière de sécurisation du foncier et le devenir du collectif dans la zone (7), considéré comme la cause du drame de la gestion des pâturages marocains (10), s'avèrent des mesures pressantes au même titre que le besoin d'harmonisation et de mise à jour des textes qui régissent directement ou indirectement l'utilisation des ressources pastorales (19).

Enfin, la relève des éleveurs de plus en plus âgés reste un défi qui nécessite de promouvoir davantage les programmes de développement humain. Des efforts de conciliation entre les parents et les jeunes ne peuvent être que bénéfiques pour une société où naissent de nouveaux conflits entre les générations du fait de l'absence de communication et de divergences des objectifs.

\section{CONCLUSION}

Il ressort que l'évolution du système pastoral se manifeste clairement par une dynamique régressive constante des ressources suite à la dégradation continue des parcours. Les stratégies suivies par les éleveurs apparaissent non adaptées à la dynamique constatée. La régulation conséquente en termes d'effectifs n'est malheureusement pas observée, donnant lieu à une pression croissante sur la ressource de base. Les facteurs anthropiques demeurent plus importants que les facteurs climatiques qui n'ont, eux, qu'un effet amplifiant les usages abusifs et opportunistes des pasteurs peu soucieux de la ressource collective. Les formes de production basées sur les déplacements qui tenaient compte de l'état des pâturages ne sont plus présentes aujourd'hui. Les analyses historiques montrent une forte tendance à la sédentarisation et à l'intensification des élevages suite à la baisse de la production en fourrage des parcours.

Malgré sa longue tradition et son adaptation à l'écosystème, l'élevage pastoral au Maroc oriental ne peut perdurer sans mesures de redressement des tendances observées. Il est capital de combiner, au niveau du plan d'intervention et du schéma directeur, des mesures de réhabilitation et d'amélioration des parcours dégradés et des modes de gestion rationnelle fondés sur l'organisation effective des usagers et des règles de pacage et de contrôle des charges animales et de la mobilité. Un système de veille biologique et d'alerte précédant la prise de décision, alimenté régulièrement par des données sur l'état des ressources pastorales, sur la dynamique et les prélèvements des troupeaux (moyennant des indicateurs de structure et de fonctionnement clés), doit être mis en place par les structures de tutelle sur le collectif et sous le contrôle d'unité de suivi et d'évaluation.

\section{BIBLIOGRAPHIE}

1. ABATE T., EBRO A., NIGATU L., 2010. Traditional rangeland resources and pastoralist's perceptions on land degradation in South-East Ethiopia. Trop. Grassl., 44: 202-212.

2. ACHERKOUK M., MAATOUGUI A., BOUAYAD A., 2006. Caractérisation économique de l'élevage ovin dans les hauts plateaux de I'Oriental. In : Boulonoir B., Paquay R., éds, L'élevage du mouton et ses systèmes de production au Maroc. Rabat, Maroc, Nadacom, p. 91-102.

3. ACHERKOUK M., MAATOUGUI A., El HOUMAIZI A., 2011 Communautés végétales et faciès pastoraux dans la zone de TaourirtTafoughalt du Maroc oriental. Ecologie et inventaire floristique. Acta Botanica Malacit., 36 : 125-136.

4. AIDOUD A., 1994. Pâturages et désertification des steppes arides en Algérie : cas de la steppe d'alfa. Paralelo, 37 : 33-42.

5. AMRI L., 1992. Mutation du système de production ovin dans les hautes steppes tunisiennes. Cas de la région de Sidi Bouzid en Tunisie centrale. In : Séminaire int. Réseau parcours, privatisation de l'espace pastoral et sédentarisation, Chlef, Algérie, 7-9 avr. 1992, 113 p.

6. BECHCHARI A., EL KOUDRIM M., CHERKANI A., 2014. La cohabitation des races ovines Ouled Jellal et Béni Guil et développement de l'élevage ovin dans le système pastoral du Maroc Oriental. In : Séminaire int. Arimnet, Mediterranean biodiversity as a tool for the sustainable development of the small ruminant sector. http://arimdomestic.net/attachments/article/134/ BECCHARI_cohabitation.pdf (accessed 03/08/2015)

7. BENARADJ A., BOUCHERIT H., 2014. Gestion intégrée des ressources pastorales dans la zone de Naâma (Algérie). Rev. Algérienne Dév. Econ., 1 : 19-29.

8. BENBRAHIM K.F., ISMAILI M., FIKRI B., TRIBAK A., 2004. Problèmes de dégradation de l'environnement par la désertification et la déforestation, impact du phénomène au Maroc. Sécheresse, 15 : $307-$ 320.

9. BERKAT O., HAMMOUMI M., 1990. Etude de synthèses cartographique sur les parcours. Notice des cartes du marché n³/89/DE. Rabat, Maroc, ministère de l'Agriculture, 56 p.

10. CHALLOT J.P., 1952. La restauration et I'amélioration des pâturages semi-arides du Maroc. Rev. Forest. Fr., 4 : 280-287.

11. CRPII, 2011. Plan de gestion écosystémique spécifique (PDGE) filière viande rouge ovine de la zone steppique des hauts plateaux de l'Oriental et de la moyenne Moulouya. In : Séminaire présentation des PDGE des filières du programme PAPSA (UE) / Plan Maroc Vert, Oujda, Maroc, Sept. 2012, 93 p.

12. DUTILLY-DIANE C., ACHERKOUK M., BECHCHARI A., BOUAYAD A., EL KOUDRIM M. MAATOUGUI A., 2007. Dominance communautaire dans I'exploitation des espaces pastoraux : impacts sur les modes de vie et implications pour la gestion des parcours du Maroc oriental. Cah. Agric., $16: 338-346$.

13. EL HARRADII, 1997. Aménagement, érosion et désertification sur les Hauts-Plateaux du Maroc oriental. Rev. Méditerr., 86 : 15-23.

14. EL MOURID M., NEFZAOUI A., 2008. Rangeland improvement and management in arid and semi-arid environment of West Asia and North Africa. Giza, Egypt, KariaNet.

15. ERES, 1970. Etude pour l'aménagement des terrains de parcours du Maroc oriental : situation actuelle. Rabat, Maroc, ministère de I'Agriculture et de la Pêche maritime, 439 p.

16. FAO, 1997. Livestock and the environment: finding balance. Rome, Italy, FAO. www.fao.org/docrep/x5303e/x5303e00 (accessed $12 / 09 / 2014)$

17. FERCHICHI A., 1999. Rangelands of pre-Saharan Tunisia: potential desertification, state and management problems. In: Etienne M., Ed. Dynamics and sustainability of Mediterranean pastoral system. Cah. Options Méditerr., 39: 137-141.

18. FIDA, 2002. Projet de développement des parcours et de l'élevage de l'Oriental. Rapport d'évaluation à mi-parcours. Paris, France, FIDA, $210 \mathrm{p}$.

19. FIDA, 2002. Projet de développement des parcours et de l'élevage de I'Oriental. Rapport d'évaluation intermédiaire. www.ifad.org/evaluation/ public_html/eksyst/doc/prj/region/pn/morocco/Morocco.pdf (consulté 16/07/2014) 
20. JAKOSY O., QUAAS M.F., BAUMGARTNER S., FRANK K., 2015. Adapting livestock management to spatio-temporal heterogeneity in semi-arid rangelands. J. Environ. Manage., 162: 179-189.

21. LANDRY A.D., DJAMEN P., VALL E., KOUSSOU M.O., COULIBALY D., LOSSOUARN J., 2012. Du nomadisme à la sédentarisation. Rev. Ethnoécol. www.ethnoecologie.revues.org/779?lang=en (consulté 10/09/2014)

22. LE HOUEROU H.N., 1995. Considérations biogéographiques sur les steppes arides du nord de l'Afrique. Sécheresse, 6 : 167-182.

23. MAHYOU H., MIMOUNI J., HADRIA R., 2010. Mise en place d'un système d'alerte précoce à la sécheresse. Oujda, Maroc, Hilalpress, 29 p.

24. NARJISSE H., EL HARIZI K., HERZENNI A., BOUAYAD A., BECHCHARI A., MAATOUGUI A., MAHYOU H., MIMOUNI J., ACHERKOUK M., EL KOUDRIM M., RAHMI M., SNAIBI W., ZIRARI M., 2005. Autonomisation des ruraux dans les hauts plateaux de l'Oriental (Maroc). Rapport de restitution. Rabat, Maroc, INRA, 170 p.

25. NDIAYE D.S., 2010. Cadre conceptuel d'analyse DPSIR /FPEIR www.fao.org/nr/lada/index.php?option=com_docman\&task=doc_downlo ad\&gid=592\&ltemid=165\&lang=fr (consulté 04/03/2014)

26. OUEDRAOGO D., 2010. Perception et adaptation des éleveurs pasteurs au changement climatique en zones sahélienne, nord et sud soudaniennes du Burkina Faso. Bobo-Dioulasso, Burkina Faso, IDR. www.beep.ird.fr/collect/upb/index/assoc/ IDR-2010-OUE-PER/IDR-2010OUE-PER.pdf (consulté 20/07/2014)

27. PDPEO, 1988. Rapport d'identification du Projet de développement des parcours et de l'élevage dans l'Oriental. Figuig, Maroc, ministère de l'Agriculture, $93 \mathrm{p}$.

\section{Summary}

Bechchari A., El Aich A., Mahyou H., Baghdad M., Bendaou M. Analysis of the evolution of the pastoral system of Eastern Morocco

The pastoral system of the high plateaus of Eastern Morocco is characterized by extensive rangelands $(3.2$ million hectares) and the dominance of sheep breeding (1.2 million head). The study was conducted at Beni Mathar in the North, and Maatarka in the South. It addresses the issues of change in rangeland use and herd management, from the hypothesis of rangeland degradation followed by a decrease in grazing pressure. Dynamics are perceived by studying land use in 1970, 1988 and 2013. A survey involving 85 breeders and participatory workshops contributed to identify flock management types. The land use analysis shows a regression of good plant formations replaced by less valuable formations, and a decrease in rangeland forage. Concomitantly, there is a rise in the number of animals that are decreasingly mobile and increasingly supplemented (mainly barley and wheat bran cover $20-40 \%$ of needs), and a change in herd composition as the local Beni Guil breed is gradually replaced by the Ouled Djellal breed for its competitive market value. The history of the studied pastoral society shows resilience despite socioeconomic and climatic disturbances recorded since independence. Ranchers (over 200 ewes) benefit more from the rangelands and marketing than small farmers (less than 50 ewes) who are more at risk to abandon farming.

Keywords: Sheep - Rangeland - Land degradation Pastoralism - Development policy - Morocco.
28. PDPEO, 2003. Rapport d'achèvement du Projet de développement des parcours et de l'élevage dans I'Oriental. Figuig, Maroc, ministère de I'Agriculture, $56 \mathrm{p}$

29. RACHIK H., 2000. Comment rester nomade. Casablanca, Maroc, Afrique Orient, $175 \mathrm{p}$.

30. REGASSA T., 2008. Famers' perception of environmental degradation and their response to environmental management. A case of Dale Woreda, Sidama zone, SNNPR. Doct. Thesis, Addis Ababa University, College of Development Studies, Institute of Population Studies, Ethiopia, $75 \mathrm{p}$.

31. TABLI M., 1997. Action anthropique et dégradation de I'environnement aride : la désertification en Tunisie du Sud-Est. Méditerranée, 86 : 25-31.

32. TAG B., 2003. Espace et société agro-pastorale en mutation dans le Maroc oriental steppique. Sais-Fès, Maroc, faculté des Lettres et des Sciences humaines, 375 p. (Sér. Thèses et Monographies, $n^{\circ} 5$ )

33. TTOBA, 2008. Etude sur l'opérationnalisation de la stratégie de développement des parcours et de l'élevage dans l'oriental : le plan directeur de l'aménagement des hauts plateaux de l'Oriental. PDPEO, FIDA, www.menarid.icarda.org/Projects/MPCDPRASA/ StudiesTechnical\%20 Background/Forms/AllItems.aspx (consulté 21/04/2015)

34. UNCCD, 1994. Acteurs institutionnels luttant contre la désertification. Comité scientifique français de la désertification. www. csf-desertification.org/international/item/unccd (consulté 24/03/2015)

Accepted 16 September 2015; Online publication 30 September 2015

\section{Resumen}

Bechchari A., El Aich A., Mahyou H., Baghdad M., Bendaou M. Análisis de la evolución del sistema pastoril de Marruecos oriental

El sistema pastoril de las Mesetas Altas de Marruecos oriental se caracteriza por la extensión del recorrido (3,2 millones de hectáreas) y la dominancia de la cría ovina (1,2 millones de cabezas). El presente trabajo, Ilevado a cabo en Beni Mathar al Norte y en Maâtarka al Sur, trata el tema de los cambios de ocupación de las tierras de recorrido y de gestión de los rebaños, a partir de la hipótesis de una degradación de los recorridos, seguida de una baja de la presión de pastoreo. Esta dinámica es percibida por el estudio de la ocupación de suelos en 1970, 1988 y 2013. Los modos de gestión son identificados mediante encuestas (85) y durante talleres participativos. El análisis de la ocupación de los suelos muestra la regresión de buenas formaciones vegetales y su substitución por formaciones de menor valor, así como una disminución de los aportes forrajeros en el recorrido. Paralelamente, se nota un aumento de los efectivos de animales cada vez menos móviles y cada vez más suplementados (20 a 40\% de satisfacción de las necesidades esencialmente mediante cebada y salvado de trigo) y un cambio progresivo en la composición racial de los rebaños a favor de la raza Ouled Djellal (con valor comercial competitivo) en detrimento de la raza local Beni Guil. La historia de la sociedad pastoril estudiada atesta de una capacidad de adaptación, a pesar de las perturbaciones socio económicas y climáticas observadas desde la independencia. Los grandes criadores (más de 200 ovejas) benefician más de los pastizales y especulan mejor con la comercialización, mientras que los pequeños criadores (menos de 50 ovejas) están más bajo la amenaza del abandono de la cría.

Palabras clave: Ovino - Tierra de pastos - Degradación de terras - Pastoralismo - Política de desarrollo - Marruecos. 